Pacific Journal of Mathematic 


\title{
PLANE GEOMETRIES FROM CONVEX PLATES
}

\author{
MarLow Sholander
}

1. Introduction. It is shown below that to each member of a general class of two-dimensional convex bodies there corresponds an affine geometry in the sense of Artin [1] and an S. L. space in the sense of Busemann [4].

A two-dimensional convex body is called a convex plate. For the few elementary properties of such plates assumed here, see [3].

Let $K$ be a convex plate, and let $K^{0}$ denote its boundary curve. All constructions are to be made in the plane $E$ of $K$. Consider an arbitrary direction $\phi$ in $E$ and the two lines of support to $K$ in this direction. Let $t_{0}$ be the line of support whose associated half-plane in the direction $\phi+\pi / 2$ contains $K$. Let $t_{1}$ be the other line of support. For $0<i<1$, let $t_{i}$ be the line parallel to $t_{0}$ which divides line segments extending from $t_{0}$ to $t_{1}$ in the ratio of $i$ to $1-i$. Let $t_{i}$ cut $K^{0}$ at points $R_{i}$ and $T_{i}$ so that the directed segment $R_{i} T_{i}$ has direction $\phi$.

For $0<i<1$ and $0<j<1$, let $S_{i j}$ be the point which divides $R_{i} T_{i}$ in the ratio of $j$ to $1-j$. The set $s_{j}=U_{i} S_{i j}$ is an open Jordan arc whose endpoints are points of contact of $t_{0}$ and $t_{1}$ with $K$. A set $s_{j}$ is called a strut. Other struts may be obtained by varying $\phi$. When the direction needs emphasis, the above notations are modified by affixing the angle in parentheses, for example, $R_{i}(\phi)$ or $s_{j}(\phi)$. Two struts with no common points or all points in common are called parallel. Clearly $s_{j}(\phi)$ and $s_{k}(\phi)$ are parallel.

Under the name Durchlinien, Zindler [6] studied struts of the form $s_{1 / 2}(\phi)$. It is easy to see that $s_{1 / 2}(\phi)$ halves the area of $K$, and that the centroid of $K$ is contained in the convex hull of this strut.

2. A preliminary theorem. This section is devoted to a proof of the following theorem. An edge of $K$ is defined as a (maximal) line segment in $K^{0}$.

THEOREM. If for distinct directions $\phi$ and $\psi$, struts $s_{i}(\phi)$ and $s_{j}(\psi)$ meet at distinct points $P$ and $Q$, they meet at all points of the segment $P Q$. Such segments of intersection occur if and only if $K$ has at least two edges.

Received May 19, 1952. A part of this paper was written while the author was under contract to the Office of Naval Research.

Pacific J. Math. 3 (1953), 667-671 
Proof. Let $i=1 /(1+a)$ and $j=1 /(1+b)$. From the affine invariant nature of the problem, we may assume $\phi$ and $\psi$ are respectively the positive $x$ - and positive $y$-directions in $E$, where $P$ has been chosen as the origin. We may assume the chords passing through $P$ along the axes are $P_{3} P_{1}$ and $P_{4} P_{2}$, where $P_{1}, P_{2}, P_{3}$, and $P_{4}$ have respectively the coordinates $(a, 0),(0, b),(-1,0)$, and $(0,-1)$. If $P_{4} P_{1}$ is parallel to $P_{3} P_{2}$, let $n$ be the line parallel to these lines which passes through $P$. Otherwise let $n$ be the line on $P$ and the point of intersection of these lines. Finally, we may assume that $Q$ lies in the first quadrant on or above the line $n$. Let $Q$ have coordinates $(r, s)$.

Let the chords through $Q$ parallel to the axes be $Q_{3} Q_{1}$ and $Q_{4} Q_{2}$. Coordinates of $Q_{1}, Q_{2}, Q_{3}$, and $Q_{4}$ have respectively the form $(r+a p, s),(r, s+b q)$, $(r-p, s)$, and $(r, s-q)$. We note that $P_{4} P_{1}, n$, and $P_{3} P_{2}$ have respectively the equations

$$
a y=x-a, a(b+1) y=b(a+1) x \text {, and } y=b x+b .
$$

Since $Q$ is on or above $n$,

$$
b(a+1) r \leqq a(b+1) s
$$

Because $K$ is convex, $Q_{2}$ cannot be above $P_{2} P_{3}$; that is,

$$
s+b q \leqq b(r+1) \text {. }
$$

Multiply (2) by $a$ and add to (1). This gives

$$
r-a \leqq a(s-q)
$$

that is, $Q_{4}$ is on or above $P_{1} P_{4}$. Moreover, equality in $(3)$ implies equality in (1) and (2).

Consider first the case $r<a$. Here, since $Q_{4}$ cannot be above $P_{1} P_{4}$, it lies on $P_{1} P_{4}$. Thus equality holds in (2), and $Q_{2}$ lies on $P_{2} P_{3}$. Since $P_{4}, Q_{4}$, and $P_{1}$ are distinct and collinear, they are on an edge of $K$. Similarly, $Q_{2}, P_{2}$, and $P_{3}$ lie on an edge.

In the case $r \geqq a$,

$$
\text { slope } P_{4} P_{1} \leqq \text { slope } Q_{4} Q_{1}, 1 / a \leqq q / a p \text {, and } p \leqq q .
$$

If $s<b, Q_{3}$ cannot be below $P_{2} P_{3}$; that is,

$$
b(r+1) \leqq s+b p
$$

Together with (2) this yields $q \leqq p$. Hence $p=q$, and equality holds in both (2) 
and (4). This shows that $Q_{2}, P_{2}, Q_{3}, P_{3}$ are collinear, and hence on an edge of $K$. Furthermore, slope $P_{4} P_{1}=$ slope $Q_{4} Q_{1}$, and $P_{4}, P_{1}, Q_{4}, Q_{1}$ are on an edge. If $s \geqq b, Q_{3}$ cannot be above $P_{2} P_{3}$, slope $Q_{3} Q_{2} \leqq$ slope $P_{3} P_{2}, b q / p \leqq b$, and $q \leqq p$. Again $p=q$, slope $P_{4} P_{1}=$ slope $Q_{4} Q_{1}$, and slope $P_{3} P_{2}=$ slope $Q_{3} Q_{2}$. An edge of $K$ contains $P_{4}, P_{1}, Q_{4}$, and $Q_{1}$, and another edge contains $Q_{2}, Q_{3}$, $P_{2}$, and $P_{3}$.

3. Affine geometries. Consider a convex plate $K$ with the properties:

(i) $K$ has at most one edge;

(ii) $K$ has no corners.

Let $I$ be the set of inner points of $K$. Consider distinct points $P$ and $Q$ in $I$. Assume, for a given $\phi$, that $P$ is on $s_{j}(\phi)$ and $Q$ is on $s_{k}(\phi), j<k$. Clearly $P$ is on $s_{1-j}(\phi+\pi)$, and $Q$ is on $s_{1-k}(\phi+\pi)$. From considerations of continuity, there exists a direction $\psi$ such that $P$ and $Q$ are on a strut $s_{i}(\psi)$. From this and from the previous section we have the following result.

Property I. Two distinct points in $I$ lie on one and only one strut.

Consider now a strut $s_{i}(\phi)$ and a point $P$ in $l$. The strut $s_{j}(\phi)$ which passes through $P$ is parallel to $s_{i}(\phi)$. On the other hand, let $s_{k}(\psi), \psi \neq \phi$, pass through $P$. Since $s_{i}(\phi)$ and $s_{k}(\psi)$ have endpoints which separate one another on $K^{0}$. these struts have some point of $I$ in common, and the following holds.

Property II. Given a strut $s$ and a point $P$ in $l$, there is one and only one strut through $P$ and parallel to $s$.

PROPERTY III. There are three points of $l$ not on a strut.

These three properties are Axioms I, II, and III of Artin [1]. Listed in Lattice theory $[2, \mathrm{p} .110]$ as APG1, APG2, and APG3, they classify $I$ as a plane affine geometry.

It would be of interest to know what sets $I$ satisfy Artin's Axiom IV (see Appendix), or even what sets have nontrivial dilatations. An ellipse $K$ yields an $I$ with all the desired properties. To show this it is sufficient to consider the case where $K$ is the circle

$$
x^{2}+y^{2} \leqq a^{2}
$$

Consider the sphere

$$
S: x^{2}+y^{2}+(z-a)^{2}=a^{2},
$$


resting on the origin of the $x y$-plane $E$. The line

$$
x \cos \phi+y \sin \phi=R
$$

in $E$ projects from the center of $S$ into a great half-circle on $S$. This half-circle projects perpendicularly on $E$ into a half-ellipse, the strut $s_{i}(\phi)$, where

$$
2 i=1+R / \sqrt{R^{2}+a^{2}} .
$$

Thus the mapping which takes $(r, \theta)$ in $l$ into the point $(R, \theta)$ of $E$, where

$$
R \sqrt{a^{2}-r^{2}}=a r,
$$

places the struts in one-to-one correspondence with straight lines. In this example, we have a finite model for Euclidean geometry.

4. Other geometries. In general we may obtain a plane projective geometry from a plane affine geometry by adjoining an ideal line (see $[2, \mathrm{p} .110])$. In this case $K^{0}$ serves as the ideal line. The affine and projective geometries associated with $K$ are examples of matroid lattices.

In $\S 3$ we mapped an elliptical $I$ onto the Euclidean plane $E$. A similar mapping may be defined for any $l$ so that struts map on curves in $E$ which satisfy the hypotheses of [4, p. 89, Th.1]. It follows that a metric may be introduced (in $E$ and hence) in $I$ which makes of $I$ an S. L. Space of Busemann: $I$ will be finitely compact, convex in the sense of Menger, externally convex in the sense of Busemann, and the struts will be geodesics under this metric. This $\mathrm{S}$. L. space also satisfies the Euclidean Parallel Axiom. In fact, all Hilbert's (plane) Axioms [5] are satisfied except the congruence axioms. The determination of the conditions under which the latter hold is an open problem.

5. Appendix. Artin's Axiom IV, not readily available to all readers, is given below after necessary introductory material. Using Axioms I-IV, we may assign coordinates $(\alpha, \beta)$ to points so that the equation of a "strut" is linear.

The set of points considered is called a plane. A mapping $\sigma$ associates with every point $P$ a point $P^{\prime}=\sigma(P)$. A mapping is called a dilatation if to each pair of points $P, Q$ correspond parallel struts $s$ and $s^{\prime}$ such that $P$ and $Q$ lie on $s$, and $P^{\prime}$ and $Q^{\prime}$ lie on $s^{\prime}$. The identity mapping of the plane is denoted by 1 . A translation is a dilatation which is either 1 or else has no fixed points. A trace of a dilatation $o$ is a strut which contains a point $P$ and its image $P^{\prime}$. (If $P \neq P^{\prime}$, there is a unique trace on $P$.) A homomorphism is a correspondence from translation $\tau$ to translation $\tau^{\alpha}$ such that each trace of $\tau$ is a trace of $\tau^{\alpha}$ and such that 


$$
\left(\tau_{1} \tau_{2}\right)^{\alpha}=\tau_{1}^{a} \cdot \tau_{2}^{\alpha}
$$

Ax Iом IVa. Given $P$ and $Q$, there exists a translation carrying $P$ into $Q$.

Ax IOM IVb. Given translations $\tau_{1}$ and $\tau_{2}$ (neither equal to 1 ) with the same traces, there exists a homomorphism $\tau^{a}$ such that $\tau_{1}^{a}=\tau_{2}$.

\section{REFERENCES}

1. E. Artin, Coordinates in affine geometry, Rep. Math. Colloq. Notre Dame, Indiana, 1940.

2. Garrett Birkhoff, Lattice theory, Amer. Math. Soc. Colloq. Publ. vol.XXV (revised), New York, 1948.

3. T. Bonnesen and W. Fenchel, Theorie der konvexen Körper (reprinted), New York, 1948.

4. Herbert Busemann, Metric methods in Finsler spaces and in the foundations of geometry, Princeton University Press, Princeton, 1942.

5. D. Hilbert, Grundlagen der Geometrie, senventh edition, Leipzig and Berlin, 1930.

6. K. Zindler, Über konvexe Gebilde II, Monatsh. Math. Phys. 31 (1921), 25-56. 



\section{PACIFIC JOURNAL OF MATHEMATICS}

\section{EDITORS}

\author{
R. M. BOEINSON \\ University of California \\ Berkeley 4, California \\ E. HewitT \\ University of Washington \\ Seattle 5 , Washington
}

\section{R. P. DILWOR TH}

California Institute of Technology

Pasadena 4, California

E. F. BECKENBACH

University of California

Los Angeles 24, California

\section{ASSOCIATE EDITORS}

$\begin{array}{ll}\text { H. BUSEMANN } & \text { P. R. HALMOS } \\ \text { HERBERT FFDERER } & \text { IIEINZ HOPF } \\ \text { MARSHALL IJALL } & \text { R. D. JAMES }\end{array}$

\author{
BøRGE JESSEN \\ PAUL LÉVY \\ GEORGE PÓLYA
}

\author{
J. J. STOKER \\ E. G. STR AUS \\ KÔSAKU YOSIDA
}

\section{SFONSORS}

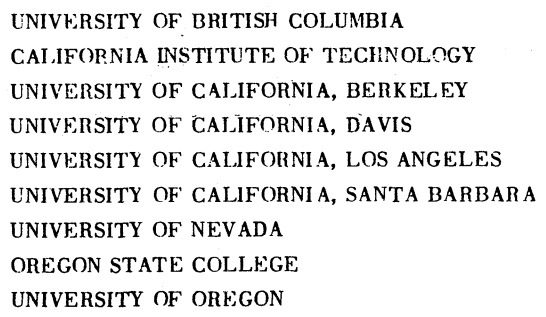

\author{
UNIVERSITY OF SOUTHERN CALIFORNIA \\ STANFORD RESEARCH INSTITUTE \\ STANFORD UNIVERSITY \\ WASHINGTON STATE COLLEGE \\ UNIVERSITY OF WASHINGTON \\ AMERICAN MATHEMATICAL SOCIETY \\ NATIONAL BUREAU OF STANDARDS, \\ INSTITUTE FOR NUMERICAL ANALYSIS
}

Mathematical papers intended for publication in the Pacific Journal of Mathematics should be typewritten (double spaced), and the author should keep a complete copy. Manuscripts may be sent to any of the editors except Robinson, whose term expires with the completion of the present volume; they might also be sent to M.M. Schiffer, Stanford University, Stanford, California, who is succeeding Robinson. All other communications to the editors should be addressed to the managing editor, E. F. Beckenbach, at the address given above.

Authors are entitled to receive 100 free reprints of their published papers and may obtain additional copies at cost.

The Pacific Journal of Mathematics is published quarterly, in March, June, September, and December. The price per volume (4 numbers) is $\$ 8.00$; single issues, $\$ 2.50$. Special price to individual faculty members of supporting institutions and to individual members of the American Mathematical Society: $\$ 4.00$ per volume; single issues, $\$ 1.25$.

Subscriptions, orders for back numbers, and changes of address should be sent to the publishers, University of California Press, Berkeley 4, California.

Printed at Ann Arbor, Michigan. Entered as second class matter at the Post Office, Berkeley, California.

\section{UNIVERSITY OF CALIFORNIA PRESS • BERKELEY AND LOS ANGELES}




\section{Pacific Journal of Mathematics}

\section{Vol. 3, No. 3 \\ May, 1953}

L. Carlitz, Some theorems on generalized Dedekind sums ............ 513

L. Carlitz, The reciprocity theorem for Dedekind sums ............. 523

Edward Richard Fadell, Identifications in singular homology theory..... . . 529

Harley M. Flanders, A method of general linear frames in Riemannian

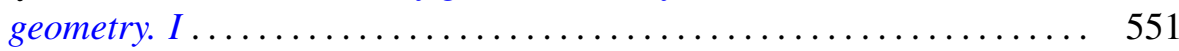

Watson Bryan Fulks, The Neumann problem for the heat equation ........ 567

Paul R. Garabedian, Orthogonal harmonic polynomials.............. 585

R. E. Greenwood and Andrew Mattei Gleason, Distribution of round-off errors for running averages .............................. 605

Arthur Eugene Livingston, The space $H^{p}, 0<p<1$, is not normable ... 613

M. N. Mikhail, On the order of the reciprocal set of a basic set of polynomials .......................................... 617

Louis Joel Mordell, On the linear independence of algebraic numbers . . . . 625

Leo Sario, Alternating method on arbitrary Riemann surfaces .......... 631

Harold Nathaniel Shapiro, Iterates of arithmetic functions and a property of the sequence of primes.............................. 647

H. Shniad, Convexity properties of integral means of analytic functions . . . . 657

Marlow C. Sholander, Plane geometries from convex plates ........... 667 